\title{
Diagnóstico del rendimiento académico de estudiantes de una escuela de educación superior en México
}

\section{Diagnosis of the academic performance of students of an undergraduate school in Mexico}

\author{
Claudia Alejandra HERNÁNDEZ HERRERA \\ Instituto Politécnico Nacional, México
}

Recibido: Marzo 2015

Evaluado: Abril 2015

Aceptado: Abril 2015

\section{Resumen}

El objetivo del artículo es realizar un diagnóstico sobre la percepción de los factores que intervienen en el rendimiento académico de los estudiantes de cinco carreras universitarias en una escuela de educación superior en México, para así reconocer las áreas de oportunidad que permitan sugerir políticas y estrategias para elevar su rendimiento. Se utilizó una muestra de 1651 estudiantes, se obtuvieron los datos a partir de un cuestionario con treinta preguntas que estudian la percepción del rendimiento académico en escala tipo Likert. Se realizó un análisis factorial exploratorio que permitiera reducir los datos, facilitar la interpretación y validar el instrumento. Se identificaron tres factores: a) el rol de los profesores, b) la evaluación y c) la motivación de los estudiantes. Se llevó a cabo un análisis comparativo por carrera. Se encontró que los estudiantes perciben que la mayoría de los maestros no se preocupan por la condición de los jóvenes en situación de reprobación. Además, casi no motivan y carecen de expresiones de sentimientos de orgullo por los logros académicos de los estudiantes. La mitad de los participantes piensa que los docentes no cubren el temario en su totalidad. Se detectó que los estudiantes poseen una alta motivación siendo esto positivo porque son alumnos dedicados y responsables. Se concluye realizando una serie de sugerencias y explicando las implicaciones que tiene este trabajo para las instituciones de educación superior.

Palabras clave: rendimiento académico, educación superior, estudiantes, evaluación, motivación del alumno.
Abstract
The objective of this article is to make a diagnosis of the perception of the factors that intervene in the academic performance of students of five university degrees in a high school in Mexico, to recognize the opportunity areas that allow suggesting policies and strategies to increase their performance. A sample of 1651 students was used; the data were obtained by a thirty-question questionnaire that studies the perception of the academic performance in the Likert scale. An exploratory factorial analysis was made to reduce the data, ease the interpretation and validate the instrument. Three factors were identified: a) the role of teachers, b) the evaluation and c) 
students' motivation. A comparative analysis of the different university degrees was made. It was found that students perceive that most of the teachers do not worry about the condition of youngsters in situation of reprobation with failure issues. Besides, teachers seldom motivate and lack the expressions of sentiments of pride towards students' academic achievements. Half of the participants think that teachers do not impart the whole syllabus. Students have a high motivation, which is positive because they prove to be responsible and dedicated. In conclusion, a series of suggestions are provided while explaining the implications that this research has for undergraduate education institutions.

Keywords: academic performance, undergraduate education, students, evaluation, students' motivation.

La educación es un elemento importante en el desarrollo socioeconómico sustentable de cualquier país, además permite que los individuos tengan acceso a mejores niveles de vida, sin embargo en México existen problemas como el rezago educativo, planes y programas obsoletos, bajo presupuesto destinado a la educación, deserción, abandono, reprobación escolar, desprofesionalización docente, bajo nivel académico de los profesores y masificación de la enseñanza (Méndez, 2012). De acuerdo con la Organización de las Naciones Unidas para la Educación la Ciencia y la Cultura (UNESCO, 2010), el acceso a la educación superior es un objetivo que se acompaña de forma simultánea con los propósitos de equidad, pertinencia y calidad.

El rendimiento académico se puede medir observando los resultados traducidos en calificaciones que se obtienen en exámenes y son notas que a lo largo del tiempo definen un número que de acuerdo con su escala obtenida puede ser positiva, regular o negativa. El analizar quiénes son los estudiantes y formas en las que aprenden permiten ayudar a las universidades a planificar acciones dirigidas para aumentar la perseverancia en estudiantes en los programas de educación superior (Cela-Ranilla, Gisbert \& De Oliveira, 2011). Por su lado, Blanz (2014) señala que el rendimiento académico es el producto de la motivación, la voluntad, la capacidad y la influencia del componente social y menciona que algunos elementos que se deben tomar en cuenta para incrementar el rendimiento son el asesoramiento de los estudiantes, mejorar las condiciones basadas en el equipamiento, asegurarse la existencia de los vínculos entre la teoría y la práctica, procurar cuidar las cargas de trabajo y darle seguimiento a los avances o retrocesos de los estudiantes. Se tiene evidencia que en diversas ocasiones la mayoría de los consejeros, profesores y especialistas que conviven con los estudiantes a menudo no saben que los jóvenes universitarios están experimentando serias dificultades académicas (Beck \& Davison, 2001). De acuerdo con Daniels, et al. (2014) y Reason, Terenzini \& Domingo (2006) las instituciones que cuidan a sus estudiantes mantienen una planeación relacionada con los factores de: a) sistemas de reclutamiento $\mathrm{y}$ de admisiones, b) interacción de los estudiantes con los profesores, c) programas que permitan involucrar a los estudiantes hacia adentro y afuera del aula, que coadyuve al desarrollo de actitudes, comportamientos y aptitudes acordes al desarrollo integral de un estudiante y d) un sistema de evaluación institucional que implique un interés en el aprendizaje del estudiante y en el aumento de la eficacia de las instituciones. 
El Instituto Politécnico Nacional (IPN) tiene una matrícula en educación superior de 103,487 alumnos, lo cuales son atendidos en 18 unidades académicas. En el año 2012 se hizo evidente el problema de la reprobación, se identificó que el $40 \%$ de los estudiantes tenían reprobadas de una a cinco asignaturas lo que llevó a reflexionar la importancia del tema del rendimiento académico y así iniciar la presente investigación. La escuela en donde se llevó a cabo el estudio cuenta con trece mil estudiantes distribuidos en cinco carreras, quienes son atendidos por novecientos profesores. La unidad académica enfrenta actualmente retos importantes los cuales debe de resolver en un futuro inmediato para así contribuir con la calidad educativa que requieren sus estudiantes y el país. El contexto que se detectó y que pueden influir en el rendimiento académico son: a) un modelo educativo con base en competencias que desde el 2004 no ha tenido una evaluación y se desconocen los impactos positivos y negativos en el rendimiento académico de los alumnos, b) planes de estudios que han permitido una sobre carga de materias; c) poca flexibilidad y c) bajas calificaciones que no permiten que los estudiantes consigan becas, entre otras.

La educación en el bachillerato de un estudiante otorga las bases sobre la cual se construye todo el futuro de su progreso académico en la universidad (Wolniak \& Engberg, 2010). Las escuelas deben de esforzarse para proporcionar los recursos que apoyen en el aprendizaje, además de trabajar en renovar el clima que se genera en las clases. (Hsu, Chiang, \& Liang, 2014). En México la enseñanza que se ofrece en este nivel es un puente importante hacia la educación superior, en el caso del Instituto Politécnico la institución cuenta con quince planteles en donde se imparte educación media superior en diversas ramas del conocimiento.

Sin embargo, el rendimiento académico es un fenómeno complejo de estudiar ya que son múltiples los factores que intervienen en el mismo. Es una realidad que la educación terciaria empodera a los jóvenes y por ello se debe de trabajar en diagnósticos que ayuden a conocer la realidad que atraviesan las escuelas para conseguir apoyar a los estudiantes con estrategias de intervención que permitan garantizar su estancia en las universidades.

\section{El rendimiento académico en la universidad}

El rendimiento académico está relacionado con elementos que interactúan continuamente con el estudiante, por un lado la institución de educación superior tiene la obligación de brindar una formación de calidad a través de los docentes, por el otro se encuentra el estudiante elemento importante del proceso de enseñanza aprendizaje y que cuenta con los argumento necesarios para identificar las acciones afirmativas implementadas que garantizan la calidad. Los indicadores son elemento que se establecen para tener el rumbo de la institución, tal es el caso de las políticas enfocadas a las tasas de graduación que pueden mejorar con un sistema e identificación temprana de estudiantes con riesgo de fracasar (Alston, Lane, \& Wright, 2014; Hyslop-Margison \& Sears, 2010; Corlett, 2005). De acuerdo con Livengood (1992) el rendimiento académico es una variable que puede ayudar a determinar si el estudiante concluirá y obtendrá su título universitario. Según An (2013) es recomendable proporcionar a los 
estudiantes una experiencia previa antes del ingreso a la universidad permite que los jóvenes experimenten el ambiente que representa este nivel educativo.

Para Torres (2014) el rendimiento académico está asociado con el conocimiento de los estilos de aprendizaje, esto permite a los profesores puedan crear un enfoque de enseñanza más centrado en el estudiante. De tal forma, que es necesario contar con profesores de calidad, que sean capaces de reconocer los estilos de aprendizaje, pero además de establecer canales de comunicación, de esta manera se pueden establecer estrategias de intervención educativa orientadas al mejora del aprendizaje de los estudiantes (Tan, Wu \& Li, 2011). Sin embargo, se debe destacar que el éxito académico es el resultado de la administración del tiempo y de las habilidades para el estudio, el apoyo de los compañeros, la familia y los profesores coadyuvan a elevar el rendimiento (Mamiseishvili, 2012).

Los autores Ruthig, et al. (2008), han evidenciado que las emociones negativas como la ansiedad y el aburrimiento predicen un bajo rendimiento académico, mientras que las emociones positivas como el disfrutar las actividades cotidianas en la universidad predicen un mejor rendimiento. Para mejorar el logro académico de los estudiantes y de inhibir la deserción, se requieren menor aburrimiento, menor ansiedad y un mayor disfrute. Estas percepciones de control académicas y emociones son en gran parte manejables tanto por los maestros y estudiantes. Según Baker (2008) los profesores deben tratar de hacer sus clases emocionalmente atractivas para fomentar la participación de los estudiantes en clase y de esta forma prevenir el aburrimiento. También se encontró que el estilo de afrontamiento académico de los estudiantes universitarios y la motivación depende del manejo de su estrés (Struthers, Perry \& Menec, 2000).

El rendimiento académico está relacionado con el fracaso escolar, éste depende de diversos factores como los sociales, escolares y físicos, cada estudiante es distinto, cada uno tiene su personalidad, su capacidad de adaptación y de acción, su bienestar y su desempeño en la escuela dependerán de sus esfuerzos y de sus elecciones. Roman (2014) reconoce que algunos estudiantes por falta de autoestima, asignan sus fracasos a causas internas y por lo tanto también son malas sus expectativas para lograr buenas calificaciones.

El rendimiento académico está conectado con el capital humano, que es considerado como un elemento determinante en el crecimiento económico y está asociado con la competitividad y la calidad de la educación superior que los países ofrecen a sus jóvenes (Baumann \& Hamin, 2011). Éste depende de diversos factores como la actitud negativa, falta de interés, escaso esfuerzo o motivación, insuficientes habilidades para la lectura y la escritura, aunado a la condición familiar y laboral de los estudiantes universitarios (Finch \& Taylor, 2013).

Además el rendimiento académico tiene una relación con la transición entre sistemas educativos, de acuerdo con Komarraju, Ramsey y Rinella (2013) algunos estudiantes universitarios tienen la capacidad y las habilidades psicosociales que permiten una transición mucho más eficaz, en otros casos el cambio provoca que los estudiantes no consigan ubicarse y por esta razón puedan poner en riesgo su estancia en 
la universidad, por lo anterior, las universidades deben afinar sus procesos de admisión de tal forma que permita seleccionar a los jóvenes que demuestren una mayor promesa para el éxito académico y profesional. Las aportaciones de Ruthig, et al. (2008) con relación a la transición señalan que las diferencias individuales en las cogniciones y las emociones juegan un papel crítico en situaciones académicas difíciles, como es el caso de la transición a la universidad, la cual es considerada como un periodo que suele estar impregnado de mucha incertidumbre.

De igual manera, la persistencia, la adaptación social y emocional y la percepción del control son elementos que intervienen de forma positiva en el rendimiento académico (Stupnisky, et al., 2007). Con respecto a la persistencia Tinto (1993) y Allen, Robbins y Oh (2008) señalan que los estudiantes que optan por carreras acordes con sus intereses son más propensos a persistir en su especialidad elegida.

Se sabe que el rendimiento académico interactúa con la motivación intrínseca, que son todos los comportamientos que provocan placer y se disfrutan, igualmente se tiene relación con la motivación extrínseca que son aquellas conductas que se llevan a cabo para lograr objetivos y así recibir recompensas, por último, la integración académica, considerada como la cantidad de energía puesta en el aprendizaje, la obtención de buenas calificaciones y las adecuadas interacciones con el profesorado (Clark, Middleton, Nguyen \& Zwick, 2014; Acee, Cho, Kim \& Weinstein, 2012; Prospero \& Vohra-Gupta, 2007).

Los investigadores Perry, Hall y Ruthing (2005) mencionan que el rendimiento académico tiene relación con la percepción académica, ésta se refiere a las creencias de los estudiantes sobre algunos atributos que creen poseer, como la capacidad intelectual, la resistencia física, estrategias de trabajo, habilidades sociales y la experiencia educativa hacen una diferencia en su rendimiento escolar. De igual forma, los estudiantes que reconocen trabajar duro y que se sienten satisfechos sobre las carreras que cursan, tienen mejores calificaciones y son más productivos en sus estudios universitarios. Por otra parte, los estudiantes que atribuyen sus bajas calificaciones a la falta de capacidad, sienten emociones que provocan vergüenza, tristeza, baja autoestima y depresión.

Según Conti (2000) el rendimiento académico se incrementa a través de las metas autónomas que promueven las motivaciones y mejoran la adaptación en la universidad. Se ha descubierto que la satisfacción con la tarea se asocia positivamente con el rendimiento académico reportando experiencias de aprendizaje y motivación (Clark, et al., 2014). Por otra parte, de acuerdo con Livengood (1992) el rendimiento académico está relacionado con la confianza en la inteligencia, de esta manera, se pueden encontrar jóvenes dispuestos a conservar sus altos promedios, tal vez castigando su aprendizaje, también existen alumnos que su único interés es aprender sin importar que tan estricto y difícil sea el profesor, para ellos no tienen relevancia las buenas calificaciones. Asimismo, Ruthig, et al. (2008), así como Fenning y May (2013) mencionan que las metas académicas de los estudiantes están influenciadas por una serie de factores personales y ambientales, teniendo mucho mayor impacto las creencias que tienen los alumnos sobre sí mismos y su nivel de autoestima. 
Las expectativas de los estudiantes son un indicador importante de éxito en el futuro académico (Bates \& Anderson, 2014). Otro elemento que se asocia de forma positiva para obtener buenas calificaciones es la satisfacción con las actividades que deben de realizar los estudiantes, éstas pueden traer consigo experiencia positivas de aprendizaje y motivación (Bembenutty \& White, 2013).

Los modelos de aprendizaje centrados en el estudiante contribuyen en el rendimiento académico y éstos deben estar basados en los principios de: a) el reconocimiento de los procesos individuales que involucran los estilos de aprendizaje de los estudiantes, b) las interacciones con compañeros e instructores y c) los efectos que tiene el diseño de los planes de estudio sobre las evaluaciones de los estudiantes (Cela-Ranilla, Gisbert \& De Oliveira, 2011).

Los estudios de Nonis y Hudson (2006) han evidenciado que las variables de motivación y el tiempo para el estudio influyen de manera significativa en el rendimiento académico, es decir, cuanto más tiempo se dedica el estudio mejor rendimiento académico se tiene, sin embargo depende del tipo de curso, el tipo de actividad de aprendizaje y la ansiedad por dominar un tema (Masui, et al., 2012). Asimismo, Davis, Winsler y Middleton (2006), indicaron que las recompensas son potencialmente positivas para el rendimiento académico, por otro lado, Yip (2012) señaló que las estrategias de aprendizaje y de estudio coadyuvan a elevar las calificaciones.

El rendimiento académico está relacionado con el estado de ánimo, los estudiantes que son optimistas muestran altos niveles de satisfacción hacia la universidad y por ende una alta autoevaluación, buenos valores y entusiasmo, mientras que los estudiantes pesimistas se caracterizan por baja satisfacción y baja autoevaluación (Yamada, 2014; Burke, Shanahan, \& Herlambang, 2014).

Bajo el anterior contexto, el objetivo del artículo es diagnosticar los elementos con mayor influencia en el rendimiento académico de los estudiantes en una escuela de educación superior del Instituto Politécnico Nacional para así desarrollar alguna política pública que coadyuve a incrementar el rendimiento académico de los estudiantes.

\section{Metodología}

La investigación se realizó en México en el mes de agosto del 2014, es un estudio transversal, se aplicó una encuesta con treinta preguntas que representan variables sobre el rendimiento académico, que fueron obtenidas de la literatura revisada. Se empleó la escala tipo Likert donde el valor de 5= Totalmente de acuerdo y $1=$ Totalmente en desacuerdo, la muestra fue de 1651 estudiantes de nivel superior de la escuela «Unidad Profesional Interdisciplinaria de Ingeniería y Ciencias Sociales y Administrativas» (UPIICSA) del Instituto Politécnico Nacional. La confiabilidad del instrumento se midió a partir del coeficiente de Alfa de Cronbach, el cual obtuvo como resultado 0.894, que indica que existe consistencia interna a través de todos los elementos, lo que significa que están correlacionados entre sí. 
El cuestionario se dividió en dos partes, en la primera se hallan las variables independientes: 1) género, 2) carrera, 3) turno, 4) semestre, 5) número de asignaturas no aprobadas, 6) tiempo de trayecto hacia la escuela, 7) trabajo, 8) tutor y 9) promedio.

\section{Contenido de la pregunta}

\section{Autores}

1. Para la elección de la carrera recibí orientación vocacional

2. Sentimiento de satisfacción por la carrera que se está estudiando.

3. La carrera que se está estudiando fue siempre la primer opción

\begin{tabular}{l}
\hline 4. Asistencia a las clases \\
\hline 5. Considero que dedico tiempo para estudiar, repasar y \\
hacer tarea \\
\hline 6. Considero que demuestro interés por la materias que \\
estoy cursando
\end{tabular}

7. Creo que demuestro dedicación en las tareas y trabajos que dejan los profesores

8. Creo que mi desempeño académico hasta este momento ha sido el adecuado

9. Tengo confianza de que los conocimientos que estoy adquiriendo me ayudarán a encontrar un empleo aceptable.

10. Siento que la formación académica que recibí en el bachillerato me ha ayudado para enfrentar la exigencia académica de la universidad.

11. Conozco el campo laboral que tiene relación con mi carrera

12. Cuando obtengo malas calificaciones considero que es

mi responsabilidad

Tabla 1. Estructura del cuestionario (Primera parte)

La segunda parte presenta los ítems en escala tipo Likert. La estructura del cuestionario se presenta en la tabla 1. La muestra se calculó sobre una población de trece mil alumnos, con un nivel de confianza del 95\% y un intervalo de confianza de 2.98 , dando como resultado para $n=986$, lo anterior significa que se superó el tamaño de la muestra. 


\section{\begin{tabular}{|l|l}
\hline Contenido de la pregunta & Autores \\
\hline
\end{tabular}}

13. Considero que mis profesores poseen una formación académica adecuada para estar frente a grupo dando clases.

14. Mis profesores desde el inicio del curso me informaron sobre los criterios de evaluación

15. Mis profesores señalan desde el inicio del curso los temas a tratar en la materia.

16. Mis profesores cubren en su totalidad sus programas de estudio de las asignaturas

17. Los exámenes que he presentado han sido desarrollados de acuerdo a los temas expuestos por el profesor.

18. Las calificaciones que he recibido hasta este momento considero que han sido justas

19. Los maestros demuestran compromiso en la formación de futuros profesionistas

20. Siento que mis profesores se sienten orgullosos de mis avances y éxitos como estudiante

21. Mis maestros siempre asisten a clase

22. Los profesores cuando saben que la mayoría del grupo está reprobando aplican estrategias que ayudan a regularizar nuestra situación.

23. Mis maestros me mantienen informado en tiempo mis calificaciones

24. Los maestros me han motivado a realizar actividades de investigación relacionadas con los temas vistos en clase

25. Conozco el reglamento de estudios de la institución por

lo tanto conozco mis derechos en caso de reprobar

asignaturas.

26. Los profesores y autoridades se preocupan por los alumnos que tienen asignaturas reprobadas.

27. Los profesores me motivan en sus clases

28. Los profesores están actualizados en el uso de las tecnologías de la información y comunicación

29. Los profesores cuando detectan falta de conocimientos relacionados con algún tema, se detienen y ofrecen una breve regularización

30. Considero que conozco las opciones que brinda la

escuela para recuperación de asignaturas

Tabla 1. Estructura del cuestionario (Segunda parte)

\section{Resultados}

Se aplicó el cuestionario a 1651 estudiantes, siendo la carrera de ingeniería industrial con el $61 \%$ los que en su mayoría participaron en el estudio, en segundo lugar la licenciatura de administración industrial con el $24 \%$, en tercer lugar ingeniería en transporte con el $12 \%$, cuarto lugar ingeniería en informática con el $2 \%$ y en quinto lugar la carrera de ciencias de la informática con el 1\%. El 43\% de los participantes 
son mujeres y el $57 \%$ hombres. Además el $50 \%$ de los estudiantes son del turno matutino y el $50 \%$ del turno vespertino. El promedio de las calificaciones se encuentra en una media del 7.67 de una escala del cero al diez, con una desviación estándar de 1.12 , se observa una disminución con respecto a la media de las calificaciones que los estudiantes declaran haber obtenido en la preparatoria el cual fue de 8.15 con una desviación estándar de 1.16. Con respecto al número de asignaturas suspensas se halló que el 55\% señaló no tener ninguna materia reprobada, sin embargo el $45 \%$ dijo tener una o más.

También se les cuestionó en donde habían realizado sus estudios de bachillerato, el $52 \%$ de los estudiantes señaló haber estado en alguno de los Centros Científicos y Tecnológicos del Instituto Politécnico Nacional, lo que significa que la tasa de absorción de los estudiantes que estudian en la escuela objeto de estudio proviene del sistema politécnico, la segunda escuela con mayor presencia son las preparatorias particulares (12\%) y los colegios de bachilleres con el $11 \%$, el resto proviene de los demás subsistemas de educación media superior. Un dato que se encontró es que sólo el $36 \%$ de los encuestados dice que cuenta con el apoyo tutorial el resto de los estudiantes no cuenta con este acompañamiento.

Se realizó un análisis factorial exploratorio por medio de la reducción de dimensiones en el software SPSS versión 20. El análisis de factores obtuvo en la prueba de Kaiser Meyer Olkin el resultado de $0.936>0.50$ lo que indica que existe una mayor grado de correlación entre los ítems y por lo tanto se pueden reducir los datos en factores. Los datos arrojaron tres factores: 1) el rol de los profesores, 2) elementos de la evaluación y 3) la motivación extrínseca del estudiante, lo anterior se obtuvo a partir de la extracción de componentes principales y rotación Varimax. En la tabla 2 se observan los resultados de la matriz de los componentes rotados, obteniéndose tres factores y de cada uno se realizaron los cálculos de sus coeficientes de alfa de Cronbach.

\begin{tabular}{llr}
\hline Factor 1 & Variable & $\begin{array}{c}\text { Correlación } \\
\text { con el factor 1 }\end{array}$ \\
\hline \multirow{4}{*}{ Profesores } & 0.757 \\
& Profesores preocupados por los estudiantes reprobados & 0.703 \\
Alfa de Cronbach & Profesores regularizan en caso de detectar falta de conocimiento & 0.679 \\
$=0.890$ & Los profesores motivan en sus clases & 0.648 \\
& Los profesores aplican estrategias para regularizar a los & \\
& estudiantes en situación de reprobación & 0.629 \\
& Los profesores están actualizados & 0.607 \\
& Profesores orgullosos de los éxitos y avances de los estudiantes & 0.593 \\
& Conocimiento sobre las opciones de recuperación de asignaturas. & 0.574 \\
& Profesores que motivan a realizar actividades de investigación & \\
& sobre sus asignaturas. & 0.574 \\
& Conocimiento del reglamento institucional & 0.526 \\
& Profesores con experiencia laboral & 0.483 \\
& Profesores que mantienen informado al estudiante sobre sus & \\
\hline
\end{tabular}

Tabla 2. Matriz de componentes rotados, factores (1) 


\begin{tabular}{clr}
\hline Factor 2 & Variable & $\begin{array}{c}\text { Correlación } \\
\text { con el factor 2 }\end{array}$ \\
\hline \multirow{2}{*}{ Evaluación } & Conocimiento sobre la forma de evaluar la asignatura & 0.704 \\
& Profesores cubren sus temarios en su totalidad & 0.676 \\
& Conocimiento sobre los temas que se tratarán en clase. & 0.651 \\
Alfa de Cronbach & Exámenes parciales elaborados a partir de lo visto en clase. & 0.648 \\
$=0.827$ & Profesores con adecuada formación para dar clase & 0.595 \\
& Calificaciones justas & 0.550 \\
& Asistencia a clase de los profesores & 0.540 \\
& Responsabilidad del estudiante por malas calificaciones & 0.459 \\
\hline
\end{tabular}

Tabla 2. Matriz de componentes rotados, factores (2)

\begin{tabular}{llr}
\hline Factor 3 & Variable & $\begin{array}{c}\text { Correlación } \\
\text { con el factor 3 }\end{array}$ \\
\hline \multirow{2}{*}{$\begin{array}{c}\text { La motivación de } \\
\text { los estudiantes y } \\
\text { el rendimiento }\end{array}$} & Responsabilidad para realizar tareas y trabajos & 0.724 \\
académico & Dedicación para estudiar y repasar & 0.685 \\
& Percepción sobre el desempeño obtenido hasta ese momento en la & 0.660 \\
Alfa de Cronbach & carrera & 0.632 \\
$=0.688$ & Asistencia a clase & 0.582 \\
& Conocimiento sobre el campo laboral & 0.456 \\
& La formación académica anterior ayuda a salir adelante en los & 0.389 \\
& estudios que se cursan actualmente & 0.352 \\
& La carrera que se está estudiando en la primer opción & 0.338 \\
& Sentimiento de satisfacción por la carrera que se está estudiando. & 0.314 \\
& Orientación vocacional para le elección de la carrera actual. & 0.300 \\
& Confianza de los conocimientos que se están adquiriendo & \\
\hline
\end{tabular}

Tabla 2. Matriz de componentes rotados, factores (3)

\section{El rol de los profesores}

En la investigación se encontró que el $30 \%$ de los encuestados perciben que los profesores se preocupan por los suspensos, aunado a que el $37 \%$ considera que los profesores tienen la capacidad de regularizar a los alumnos en caso de detectar falta de conocimiento en los temas vistos en clase, también el $36 \%$ de los encuestados piensa que sus académicos aplican estrategias para regularizar situaciones de riesgo de reprobación. Además, se halló que sólo el $42 \%$ de los participantes percibe que sus profesores motivan en sus clases, asimismo el $37 \%$ siente que sus maestros se sienten orgullosos de sus éxitos académicos. Se encontró, que el $44 \%$ de los jóvenes percibe que sus profesores se encuentran actualizados y el 54\% reconoce que los académicos los incentivan para investigar más sobre sus materias, pero sólo el $59 \%$ de los estudiantes admite que sus maestros cuentan con la experiencia del campo laboral. Se encontró fue que sólo el $51 \%$ de los alumnos conoce el reglamento y las opciones en caso de tener problemas por reprobar asignaturas, por lo anterior, el $60 \%$ reconoce identificar las alternativas en caso de tener problemas por reprobar una o más asignaturas. 
Los resultados de las medias arrojaron que los elementos que califican a los profesores se encontraron que la carrera en ingeniería industrial es la que evalúa bajo el rol de los docentes y la carrera en ciencias de la informática percibe de mejor manera la actuación de ellos (Ver Gráfico 1).

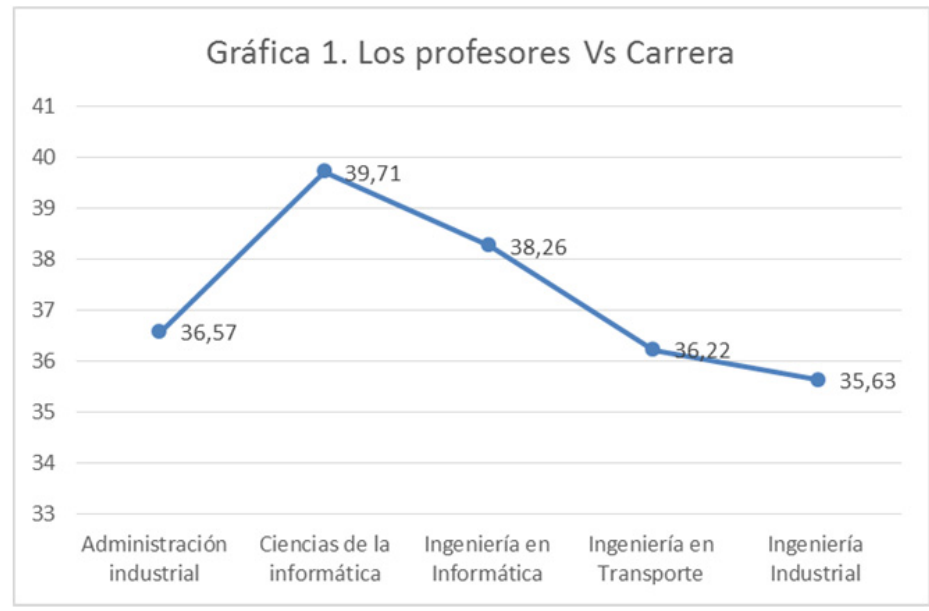

Gráfico 1. Los profesores vs. carrera

\section{La evaluación}

El 67\% de los jóvenes afirmaron conocer las formas de evaluar de los profesores, sin embargo sólo el $47 \%$ de los alumnos piensan que los académicos cubren el temario en su totalidad, además de que el $50 \%$ señalan que desde un inicio del curso los docentes les proporcionaron la lista de los temas que se verán durante el curso. A su vez, se encontró que el $64 \%$ de los jóvenes manifiesta que sus exámenes son elaborados de acuerdo con los visto en clase. Se halló que el $48 \%$ manifiesta que sus profesores poseen una formación adecuada para dar clase, además de que el 50\% considera que sus profesores asisten de forma puntual a las clases. Por otro lado, el $60 \%$ considera que las calificaciones que recibe son justas.

La carrera que mejor percibe la dimensión que integra algunos elementos de la evaluación es la carrera en ciencias de la informática y la más baja fue la carrera de ingeniería industrial (Ver gráfico 2). 


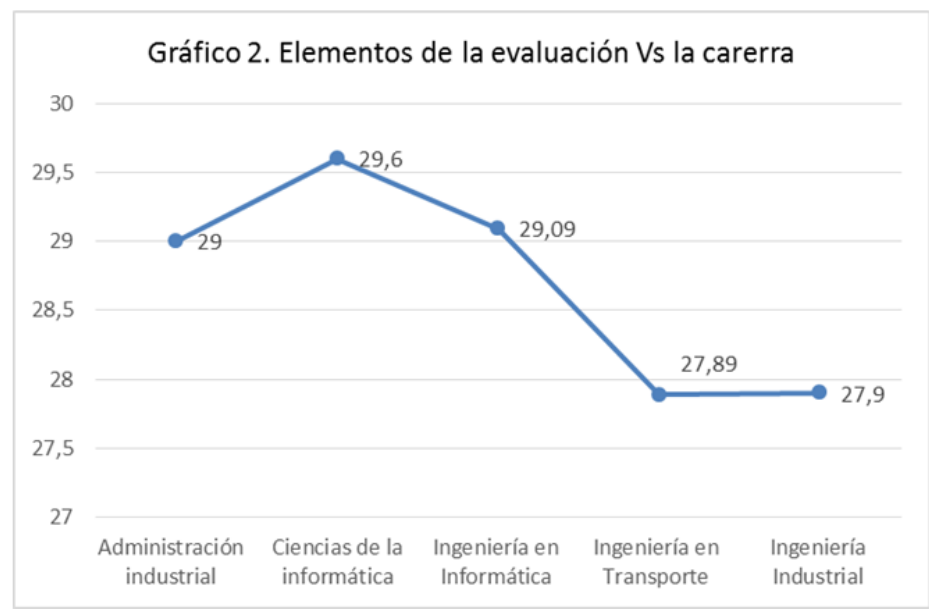

Gráfico 2. Elementos de la evaluación vs. carrera

\section{La motivación de los estudiantes y el rendimiento académico}

Se encontró que el $85 \%$ de los participantes afirman que demuestran responsabilidad con sus tareas y trabajo, además el $90 \%$ de los jóvenes manifiestan tener interés por las materias. Además, el $70 \%$ de los alumnos percibe que se dedica a estudiar y repasar, el $58 \%$ piensa que se desempeña de forma adecuada para las exigencias de la vida académica de una carrera universitaria, aunado a que el $92 \%$ dijo que procura asistir siempre a sus clases. Por otro lado, el $67 \%$ refiere que la formación adquirida en el bachillerato le ayuda a enfrentar los retos actuales que le imponen la universidad, además el $57 \%$ de los alumnos afirma que recibió orientación para la elección de la carrera. E1 $87 \%$ de los encuestados señala sentir satisfacción por la carrera que está estudiando, esto a pesar de que el 33\% señaló que está estudiando una carrera que no fue su primer opción. Realizando el cruce de variables se encontró que no se tiene una diferencia significativa entre los grupos formados de la variable carrera, sin embargo analizando las distancias de las medias se encontró que las carreras de ingeniería en informática e ingeniería en transporte tienen alumnos que se sienten más motivados en comparación con las demás licenciaturas (Ver gráfico 3). 


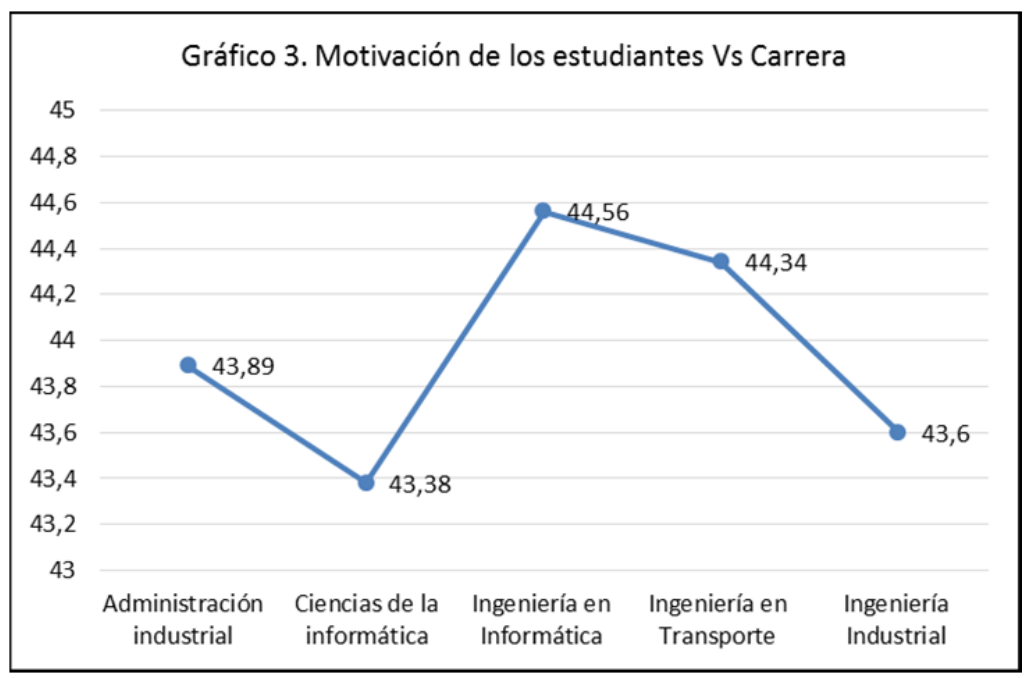

Gráfico 3. Motivación de los estudiantes vs. carrera

\section{Conclusiones}

El estudiar el rendimiento académico en una escuela de educación superior del Instituto Politécnico Nacional, ayudó a visualizar diversos escenarios que son necesarios atender. El cuestionario obtuvo tres factores principales: profesores, evaluación y motivación de los estudiantes. Los resultados obtenidos hallaron la necesidad de llevar a la reflexión el tema del rendimiento en donde las autoridades encargadas de la gestión estratégica deben desarrollar un plan maestro en donde se vislumbre una mejora a corto plazo que logre apoyar a los estudiantes que tienen problemas académicos.

Los profesores representan otra de las piezas claves en el rendimiento académico y por lo tanto son ellos los responsables de la gestión académica, en ésta se refleja la planeación de tal forma que los estudiantes conocen la manera de evaluar, aunado a la motivación que ellos pueden proporcionar a los estudiantes siendo ésta necesaria para afrontar el difícil camino que representa el iniciar y concluir una carrera universitaria. Lo anterior, permite incrementar la retención de los estudiantes y los respalda cuando presentan dificultades académicas de tal forma que por medio del acompañamiento ellos logren sus metas académicas y terminen sus estudios pero llevándose consigo el título universitario (Nonis \& Hudson, 2006).

Se sugiere que los profesores ofrezcan a los estudiantes estímulos positivos para aumentar la motivación para aprender, por ello se debe trabajar en incrementar el autoconcepto académico que tienen los estudiantes de la eficacia en el desempeño de diversas actividades, es evidente que el reconocimiento del auto-aprendizaje es fundamental para la adquisición de habilidades (Chen, Chiu \& Wang, 2014). Otro elemento de mejora es la motivación con la que trabajan los docentes en sus clases, por 
lo que se recomienda desarrollar y refinar estrategias de motivación, es bueno identificar qué recompensas estimulan a los jóvenes.

Además, se recomienda que los profesores organicen sus cursos de tal forma que sean predecibles, es decir que los temarios sean detallados desde el inicio del curso, lo anterior acompañado de una programación adecuada con fechas establecidas, criterios de evaluación, técnicas de estudio, número de trabajos que deberán de ser entregados, sugerencia en técnicas de estudio y apoyo con material didáctico complementario (Stupnisky, et al., 2007). A su vez, se propone la implementación de un procedimiento de evaluación de los profesores, éste deberá de ser capaz de diagnostica puntos que se pueden mejorar (Weinberg, Hashimoto \& Fleisher, 2009). Por último, si los profesores realmente desean mejorar el aprendizaje de los alumnos tienen que utilizar métodos de evaluación que se centren explícitamente en los procesos de aprendizaje, reconociendo que cada uno de los jóvenes aprende en diferentes ritmos, además de considerar que la evaluación no es un proceso lineal y por su complejidad es necesario revisarlo continuamente (Nygaard \& Belluigi, 2011).

La motivación es un factor importante en el éxito académico de los estudiantes, en la investigación se encontró que un poco más de la mitad de los jóvenes que participaron en el estudio reconocen que su formación académica les ha ayudado para enfrentar las exigencias. Se realizó un cruce de variables se detectó que la mitad de los jóvenes que ingresan a la unidad académica estudiaron el sistema de bachillerato que pertenece al Instituto Politécnico Nacional y en su mayoría perciben que su formación les apoya para salir adelante, sin embargo existe la otra mitad de los jóvenes que estudiaron en otros sistemas, se descubrió que los alumnos que cursaron el colegio de bachilleres y la preparatoria particular no perciben que la educación alcanzada les permita defenderse, por lo tanto se debe de tener especial cuidado y brindar más apoyo a los jóvenes que provienen de estos subsistemas.

Los jóvenes que asisten a la universidad y que formaron parte de la muestra productora de datos, son alumnos que poseen una motivación intrínseca alta, son responsables con sus tareas, muestran interés por las asignaturas y siempre asisten a clase, esto es lo que les permite salir adelante, aunado a la voluntad y el deseo de conseguir un título universitario y así llevar progreso a sus familias.

El Instituto Politécnico Nacional tiene una misión importante ante la sociedad que es formar recursos humanos capaces de brindar conocimientos y que éste contribuya al desarrollo social y económico, sin embargo la sobrecarga que está generando el ampliar el número de lugares está llevando consigo que por la cantidad de estudiantes en ocasiones no se logren detectar a tiempo los casos de fracaso académico. Lo anterior, lleva a proponer un sistema de alerta temprana que brinde con oportunidad información de los estudiantes que están presentando obstáculos para mantener su estatus de regular en la escuela, esto se debe de trabajar a la brevedad ya que el reglamento de estudios del Instituto no ofrece muchas oportunidades para regularizarse, por lo tanto, los estudiantes deben de conocer a detalle los problemas a los que se podría enfrentar en caso de presentar desfases. 
Las implicaciones para las instituciones de educación superior se concentran en la implementación de evaluaciones que permitan conocer la percepción de los estudiantes sobre su rendimiento académico y la forma en la que los actores involucrados apoyan o en su caso entrampan el proceso, por otro lado, el fortalecimiento de los programas de tutorías con profesores a los que se les pueda asignar un número de horas adecuado para la atención de los estudiantes, fortalecimiento del programa institucional de tutorías, además de informar a la comunidad académica sobre los índices de eficiencia terminal, promover la orientación vocacional, plantear la posibilidad de tener un semestre de preparación antes de ingresar a la escuela, ya que esto ayudará a que los estudiantes reconozcan el ambiente y se adapten más rápido, reforzar los cursos de inducción con explicaciones detallas sobre la importancia de mantener un adecuado apropiado rendimiento, incrementar la comunicación de las autoridades de la universidad con los padres de familia y por último el desarrollar un sistema capaz de establecer la trazabilidad de los estudiantes.

Por último, las limitaciones se observan en la participación más activa de otras carreras para poder diagnosticar lo que sucede en ellas, el cuestionario carece de preguntas abiertas que permitan recabar el sentir de los estudiantes y se vislumbra ampliar el estudio a los profesores.

\section{Agradecimientos}

Nuestro agradecimiento a la Unidad Profesional Interdisciplinaria de Ingeniería y Ciencias Sociales y Administrativas y la Secretaria de Investigación y Posgrado del Instituto Politécnico Nacional por el apoyo brindado para la realización de la investigación.

\section{Referencias bibliográficas}

ACEE, T. W., CHO, Y., KIM, J. I., \& WEINSTEIN, C. E. (2012). Educational Psychology, 32(6), 681-698.

ALLEN, J., \& ROBBINS, S. B. (2008). Prediction of college major persistence based on vocational interests, academic preparation, and first-year academic performance. Research in Higher Education, 49(1), 62-79.

ALLEN, J., ROBBINS, S. B., CASILLAS, A., \& OH, I. S. (2008). Third-year college retention and transfer: Effects of academic performance, motivation, and social connectedness. Research in Higher Education, 49(7), 647-664.

ALSTON, G. L., LANE, D., \& WRIGHT, N. J. (2014). The methodology for the early identification of students at risk for failure in a professional degree program. Currents in Pharmacy Teaching and Learning.

AN, B. P. (2013). The influence of dual enrollment on academic performance and college readiness: Differences by socioeconomic status. Research in Higher Education, 54(4), 407-432. 
BAKER, C. N. (2008). Under-represented college students and extracurricular involvement: The effects of various student organizations on academic performance. Social Psychology of Education, 11(3), 273-298.

BAKER, C. N. (2013). Social Support and Success in Higher Education: The Influence of On-Campus Support on African American and Latino College Students. The Urban Review, 45(5), 632-650.

BATES, L. A., \& ANDERSON JR, P. D. (2014). Do Expectations Make the Difference? A Look at the Effect of Educational Expectations and Academic Performance on Enrollment in Post-Secondary Education. Race and Social Problems, 6(3), 249-261.

BAUMANN, C., \& HAMIN. (2011). The role of culture, competitiveness and economic performance in explaining academic performance: a global market analysis for international student segmentation. Journal of Marketing for Higher Education, 21(2), 181-201.

BECK, H. P., \& DAVIDSON, W. D. (2001). Establishing an early warning system: Predicting low grades in college students from survey of academic orientations scores. Research in Higher Education, 42(6), 709-723.

BEMBENUTTY, H., \& WHITE, M. C. (2013). Academic performance and satisfaction with homework completion among college students. Learning and Individual Differences, 24, 83-88.

BLANZ, M. (2014). How do study satisfaction and academic performance interrelate? An investigation with students of Social Work programs. European Journal of Social Work, 17(2), 281-292.

BURKE, A., SHANAHAN, C., \& HERLAMBANG, E. (2014). An Exploratory Study Comparing Goal-Oriented Mental Imagery with Daily To-Do Lists: Supporting College Student Success. Current Psychology, 33(1), 20-34.

CELA-RANILLA, J. M., GISBERT, M., \& DE OLIVEIRA, J. M. (2011). Exploring the relationship among learning patterns, personality traits, and academic performance in freshmen. Educational Research and Evaluation, 17(3), 175-192.

CHEN, B. H., CHIU, W. C., \& WANG, C. C. (2014). The Relationship Among Academic Self-concept, Learning Strategies, and Academic Achievement: A Case Study of National Vocational College Students in Taiwan via SEM. The AsiaPacific Education Researcher, 1-13.

COLE, J. S. (2014). A survey of college-bound high school graduates regarding circadian preference, caffeine use, and academic performance. Sleep and Breathing, $1-5$.

CLARK, M. H., MIDDLETON, S. C., NGUYEN, D., \& ZWICK, L. K. (2014). Mediating relationships between academic motivation, academic integration and academic performance. Learning and Individual Differences. 
CONTI, R. (2000). College goals: Do self-determined and carefully considered goals predict intrinsic motivation, academic performance, and adjustment during the first semester?. Social Psychology of Education, 4(2), 189-211.

CORLETT, J. A. (2005). The good professor. Journal of Academic Ethics, 3(1), 27-54.

COVINGTON, M. V. (2007). A motivational analysis of academic life in college. InThe scholarship of teaching and learning in higher education: An evidence-based perspective (pp. 661-729). Springer Netherlands.

DANIELS, L. M., PERRY, R. P., STUPNISKY, R. H., STEWART, T. L., NEWALL, N. E., \& CLIFTON, R. A. (2014). The longitudinal effects of achievement goals and perceived control on university student achievement. European Journal of Psychology of Education, 29(2), 175-194.

DAVIS, K. D., WINSLER, A., \& MIDDLETON, M. (2006). Students' perceptions of rewards for academic performance by parents and teachers: Relations with achievement and motivation in college. The Journal of genetic psychology, 167(2), 211-220.

ELIASSON, A. H., LETTIERI, C. J., \& ELIASSON, A. H. (2010). Early to bed, early to rise! Sleep habits and academic performance in college students. Sleep and Breathing, 14(1), 71-75.

FENNING, B. E., \& MAY, L. N. (2013). "Where there is a will, there is an A": examining the roles of self-efficacy and self-concept in college students' current educational attainment and career planning. Social Psychology of Education, 16(4), 635-650.

FINCH, J., \& TAYLOR, I. (2013). Failure to Fail? Practice Educators' Emotional Experiences of Assessing Failing Social Work Students. Social Work Education, $32(2), 244-258$.

HSU, M. C., CHIANG, C., \& LIANG, C. (2014). The mediator effects of imagination between learning environment and academic performance: a comparison between science and engineering majors. International Journal of Technology and Design Education, 1-18.

HYSLOP-MARGISON, E. J., \& SEARS, A. M. (2010). Enhancing teacher performance: The role of professional autonomy. Interchange, 41(1), 1-15.

KOMARRAJU, M., RAMSEY, A., \& RINELLA, V. (2013). Cognitive and noncognitive predictors of college readiness and performance: Role of academic discipline. Learning and Individual Differences, 24, 103-109.

KÖSTERELİĞLU, M. A., \& KÖSTERELİOĞLU, İ. (2015). Effects of high school students' perceptions of school life quality on their academic motivation levels. Educational Research and, 274.

LIVENGOOD, J. M. (1992). Students' motivational goals and beliefs about effort and ability as they relate to college academic success. Research in Higher Education, $33(2), 247-261$. 
MAMISEISHVILI, K. (2012). International student persistence in US postsecondary institutions. Higher Education, 64(1), 1-17.

MASUI, C., BROECKMANS, J., DOUMEN, S., GROENEN, A., \& MOLENBERGHS, G. (2012). Do diligent students perform better? Complex relations between student and course characteristics, study time, and academic performance in higher education. Studies in Higher Education, (ahead-of-print), 123.

MÉNDEZ, J. (2012). Problemas económico de México y sustentabilidad, séptima edición, McGraw Hill, México.

NYGAARD, C., \& BELLUIGI, D. Z. (2011). A proposed methodology for contextualised evaluation in higher education. Assessment \& Evaluation in Higher Education, 36(6), 657-671.

NONIS, S. A., \& HUDSON, G. I. (2006). Academic performance of college students: Influence of time spent studying and working. Journal of Education for Business, 81(3), 151-159.

ORGANIZACIÓN DE LAS NACIONES UNIDAS PARA LA EDUCACIÓN LA CIENCIA Y LA CULTURA (2010). Conferencia Mundial sobre la Educación Superior 2009: La nueva dinámica de la educación superior y la investigación para el cambio social y el desarrollo (sede UNESCO, París, 5-8 de julio de 2009), Francia.

PARK, E. L., \& CHOI, B. K. (2014). Transformation of classroom spaces: traditional versus active learning classroom in colleges. Higher Education, 1-23.

PERRY, R. P., HALL, N. C., \& RUTHIG, J. C. (2005). Perceived (academic) control and scholastic attainment in higher education. In Higher education: Handbook of theory and research (pp. 363-436). Springer Netherlands.

PINTRICH, P. R., \& ZUSHO, A. (2007). Student motivation and self-regulated learning in the college classroom. In The scholarship of teaching and learning in higher education: An evidence-based perspective (pp. 731-810). Springer Netherlands.

PLANT, E. A., ERICSSON, K. A., HILL, L., \& ASBERG, K. (2005). Why study time does not predict grade point average across college students: Implications of deliberate practice for academic performance. Contemporary Educational Psychology, 30(1), 96-116.

PROSPERO, M., \& VOHRA-GUPTA, S. (2007). First generation college students: Motivation, integration, and academic achievement. Community College Journal of Research and Practice, 31(12), 963-975.

REASON, R. D., TERENZINI, P. T., \& DOMINGO, R. J. (2006). First Things First: Developing Academic Competence in the First Year of College*. Research in Higher Education, 47(2), 149-175. 
ROMAN, M. D. (2014). Students' Failure in Academic Environment. Procedia-Social and Behavioral Sciences, 114, 170-177.

RUThig, J. C., PERRY, R. P., HLADKYJ, S., HALl, N. C., PEKRUN, R., \& CHIPPERFIELD, J. G. (2008). Perceived control and emotions: Interactive effects on performance in achievement settings. Social Psychology of Education, 11(2), 161-180.

STRUTHERS, C. W., PERRY, R. P., \& MENEC, V. H. (2000). An examination of the relationship among academic stress, coping, motivation, and performance in college. Research in higher education, 41(5), 581-592.

STUPNISKY, R. H., RENAUD, R. D., PERRY, R. P., RUTHIG, J. C., HAYNES, T. L., \& CLIFTON, R. A. (2007). Comparing self-esteem and perceived control as predictors of first-year college students' academic achievement. Social Psychology of Education, 10(3), 303-330.

TAN, J., WU, Y., \& LI, Y. (2011). Research on Academic and Performance of Teachers in Universities. In Information Computing and Applications (pp. 360365). Springer Berlin Heidelberg.

TAYLOR, D. J., VATTHAUER, K. E., BRAMOWETH, A. D., RUGGERO, C., \& ROANE, B. (2013). The role of sleep in predicting college academic performance: Is it a unique predictor?. Behavioral sleep medicine, 11(3), 159-172.

TINTO, V. (1993). Leaving college: Rethinking the causes and cures of student attrition. Chicago: University of Chicago Press.

TORRES, S. M. (2014). The relationship between Latino students' learning styles and their academic performance. Community College Journal of Research and Practice,38(4), 357-369.

WOLNIAK, G. C., \& ENGBERG, M. E. (2010). Academic achievement in the first year of college: Evidence of the pervasive effects of the high school context. Research in Higher Education, 51(5), 451-467.

YAMADA, R. (2014). Learning Outcomes Among College Students in Japan: Comparative Analysis Between and Within Universities. In Measuring Quality of Undergraduate Education in Japan (pp. 97-114). Springer Singapore.

YIP, M. C. (2012). Learning strategies and self-efficacy as predictors of academic performance: a preliminary study. Quality in Higher Education, 18(1), 23-34.

WEINBERG, B. A., HASHIMOTO, M., \& FLEISHER, B. M. (2009). Evaluating teaching in higher education. The Journal of Economic Education, 40(3), 227-261. 


\section{Correspondencia con los autores}

Claudia Alejandra HERNÁNDEZ HERRERA

Av. Té \#950 esquina Resina

Col. Granjas México

C.P. 08400, Del. Iztacalco, Ciudad de México

México.

Teléfono: 56242000 EXT. 70113

e-mail: a19505@gmail.com 